\title{
PIV MEASUREMENTS OF EXIT FLOW FIELD OF CENTRIFUGAL FANS WITH CONDITIONAL SAMPLING
}

\section{Shun-Chang Yen}

Associate Professor, Department of Mechanical and Mechatronic Engineering, National Taiwan Ocean University., scyen@mail.ntou.edu.tw

Jung-Hsuan Liu

Undergraduate Student, Department of Mechanical and Mechatronic Engineering, National Taiwan Ocean University

Follow this and additional works at: https://jmstt.ntou.edu.tw/journal

Part of the Mechanical Engineering Commons

\section{Recommended Citation}

Yen, Shun-Chang and Liu, Jung-Hsuan (2007) "PIV MEASUREMENTS OF EXIT FLOW FIELD OF CENTRIFUGAL FANS WITH CONDITIONAL SAMPLING," Journal of Marine Science and Technology. Vol. 15: Iss. 3, Article 8.

DOI: $10.51400 / 2709-6998.2396$

Available at: https://jmstt.ntou.edu.tw/journal/vol15/iss3/8

This Research Article is brought to you for free and open access by Journal of Marine Science and Technology. It has been accepted for inclusion in Journal of Marine Science and Technology by an authorized editor of Journal of Marine Science and Technology. 


\title{
PIV MEASUREMENTS OF EXIT FLOW FIELD OF CENTRIFUGAL FANS WITH CONDITIONAL SAMPLING
}

\author{
Shun-Chang Yen* and Jung-Hsuan Liu**
}

Key words: centrifugal fan, PIV, fan performance.

\begin{abstract}
In this research, we have experimented with two centrifugal fans with the same geometrical configuration. These two fans are designed to rotate at different speeds driven with the same voltage. The Particle Image Velocimetry (PIV) method is applied to measure the instantaneous flow fields at the near-exit region. The AMCA 210 wind tunnel is used to find the efficiency and the performance coefficients. The velocity vectors, streamlines, vorticity contours, velocity distributions, flow rate, and performances are also presented and discussed. We find that higher rotation speed yields better efficiency.
\end{abstract}

\section{INTRODUCTION}

Centrifugal fans are used widely in industry, especially in notebook computer cooling systems. The rotating impellers of the centrifugal fan inhale the hot air flow, subsequently removing it radially along the volute. Researches on centrifugal fans were initiated by Eck [5]. He defined many geometric parameters and conducted a series of systematic analyses. These works have promoted the capability to evaluate fan performances. The coupled theories of the fan and duct designs of an air moving system were developed by Wallis [16]. Later, Bleier [1] explained these concepts and presented sample calculations to enable engineers and non-engineers to design fans that can achieve the desired criteria such as air volume, static pressure, brake horsepower, and efficiency.

The air flow turns 90 degrees due to the flow channel design. This causes many complex flow phenomena such as separation, recirculation, and reversed flow. In 1981, Raj and Swim [12] applied the flow visualization method to investigate the internal flow of

Paper Submitted 06/19/06, Accepted 11/15/06. Author for Correspondence: S.C.Yen.E-mail: scyen@mail.ntou.edu.tw.

*Associate Professor, Department of Mechanical and Mechatronic Engineering, National Taiwan Ocean University.

**Undergraduate Student, Department of Mechanical and Mechatronic Engineering, National Taiwan Ocean University. the forward-curved centrifugal fan. They found that the flow separates on the suction surface, while it moves smoothly on the pressure surface. Furthermore, the distribution of static pressure is not uniform on the impellers. Chen et al. [3] used hot wire anemometers and pressure transducers to measure the rotating stall and the inlet vortex with the inlet vane control. They found that the inlet vortex maintains a linear relationship between its frequency and the flow rate at a given vane angle. Sandra et al. [13] did an experimental investigation in a forward-curved blade centrifugal fan and found an unstable performance curve. This study presented the test performance curves revealing the unstable characteristics of the fan over a wide flow rate range. Tsurusaki et al. [15] used particle tracking velocimetry (PTV) to study the internal flow velocity distributions of a cross-flow fan in a rectangular water tank. They found that the internal flow of the cross-flow fan is disturbed strongly by the eccentric vortex generated in the cross-flow fan.

In addition, many researchers used the AMCA wind tunnel or numerical methods to analyze the exhaust efficiency of the centrifugal fan. The performance criteria are the head, flow coefficient, power coefficient, and fan efficiency. Lin and Huang [9] investigated the NACA 4412 aerofoil blade with a small forward-curved centrifugal fan by using the design concept, experimental verification, and computational simulation. The experimental results indicated that the maximum flow rate is about $1.7 \mathrm{cfm}$ for all blade inlet angles with a rotation speed of $5000 \mathrm{rpm}$. They also found that the maximum static pressure is negatively correlative with the blade inlet angle. Dilin et al. [4] studied low-pressure rotation fans experimentally and computationally to provide a satisfactory means to assess the internal flow structure and performance. They also found that $50 \%$ of pressure rise downstream of the impeller can occur across the collecting volute. Tsurusaki et al. [14] used the cross-flow fan and found that the head coefficient in the through-flow region is larger than that in the recirculating region at a high flow rate. They also found that the eccentric vortex transfers 
its kinetic energy to the through-flow and that the transferred energy increases as the flow rate decreases. Jeon [6] used a numerical method to study the characteristics of the acoustic pressure in the centrifugal impeller and wedge system. He summarized three important factors: (1) As the cut-off distance is varied from 0.0028 to $0.028 \mathrm{~m}$, the theoretical head pressure with no loss term slowly increases and (2) As the flow rate coefficient increases from 0.112 to 0.14 , the head pressure decreases.

In this paper, we use the PIV method to measure the complex centrifugal fan flow field on two different planes in the near-exit region. To design the centrifugal fans efficiently and optimally, the flow structures, velocity distributions, and flow rates are recorded and derived. Also, the performance coefficients at different flow rates are calculated and compared by using the AMCA wind tunnel test.

\section{EXPERIMENTAL ARRANGEMENTS}

\section{Experimental setup}

Figure 1 shows the experimental setup used in the present work. The dimensions of the aluminum-alloy loading platform are $50 \times 30 \times 2 \mathrm{~cm}$. Four supporting air cushions are used to eliminate vibration when these fans operate. The four stand aluminum pillars of the particle supplier are fixed on this platform. To scatter the pulse laser beam, polyamide (PM) particles are seeded into the four particle supply tubes and then spread throughout the chamber by the four cyclone particle generators. The diameters of these particles range from 35 to $70 \mu \mathrm{m}$, and the specific weight is 1.03 at $25^{\circ} \mathrm{C}$. Ignoring the effect of turbulent diffusion, the relaxation time constant is estimated to be less than $6.25 \times 10^{-5}$ seconds, and the Stokes number is at the order of $10^{-6}$. Thus, the slip between the flow and particles can be neglected in this experiment. According to Mei's method [10], the seeded particles are estimated to be capable of fluctuating up to $3 \mathrm{kHz}$. A piece of balsam wood with a reflector stuck to it is attached to the hub of the fan. A diffusionscan type Yamatake-Honeywell photoelectric switch is used to detect the impeller angle by sensing the reflected signal from the reflector. The photoelectric switch consists of a micro-diameter optical fiber infrared light transceiver (model HPF-D018) and a high gain electric amplifier (model HPX-F1). The $0.75 \mathrm{~mm}$ diameter transceiver produces a beam of modulated infrared light and receives the target-reflected optical signals. The maximum sensing distance and minimum target size are $15 \mathrm{~mm}$ and $0.2 \mathrm{~mm}$, respectively. The photo-

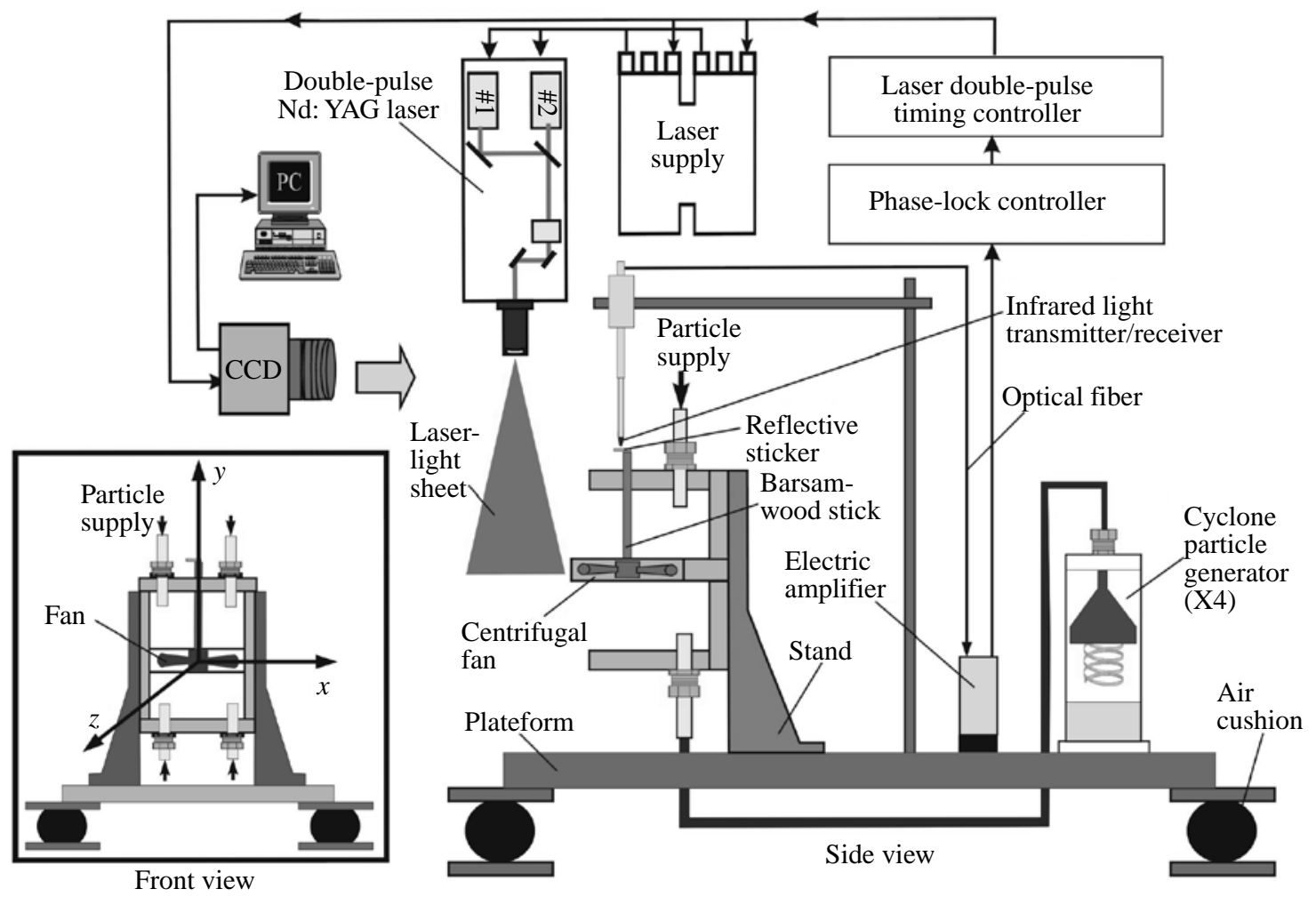

Fig. 1. Experimental setup. 
electric amplifier has a frequency response limit of 20 $\mathrm{kHz}$. By shunting with a $1.2 \mathrm{k} \Omega$ resistor, the output currents are converted into almost square-wave voltage signals. Then the output voltages are fed into a phaselock controller in the PIV system.

\section{Flow field measurements}

The PIV image acquisition system is composed of a double-pulse Nd:YAG laser, a CCD camera, and an electronic synchronizer comprising a phase-lock and laser double-pulse timing controller. As shown in Figure 1, two independent controllable pulsing lasers are packed into one casing to generate the consecutive double-pulses. The laser employs a flash lamp-pumped $\mathrm{Nd}$ :YAG rod in a thermally compensated resonator to generate radiation at $1064 \mathrm{~nm}$ via an IR head. The maximum pulsing rate is 30 double-pulses per second. The maximum pulsing energy is $90 \mathrm{~mJ}$. The pulse width is between 3-5 ns. Jittering is $\pm 0.5 \mathrm{~ns}$. The green light beams, at $532 \mathrm{~nm}$ leaving the laser head, are expanded into a 20 degree light sheet through a laser-light sheet generator which is installed at the exit of the laser-head. The thickness of the laser-light sheet is adjusted to about $0.5 \mathrm{~mm}$. For image grabbing, the particle images are recorded by a Roper Scientific Megaplus Model ES 1.0 CCD camera with a resolution of $1008 \times 1018$ pixels. The pixels are 9 microns square and can be operated continuously or in an external trigger mode. The pixel array is zoomed and mapped to a physical region at approximately $90 \mathrm{~mm}$ so that the spatial resolution is about $90 \mu \mathrm{m} /$ pixel. The external trigger mode is applied in this study by using a timing-controlled trigger signal from the electronic controller. The camera has an output channel data rate of $20 \mathrm{MHz}$, which gives a maximum frame rate of 30 frames per second. Exposure times of 0.125 to 33 milliseconds are possible with the camera's electronic shutter. When operated in the frame straddling mode, the maximum framing rate becomes 15 pairs per second. In this study, each image occupies about $2 \mathrm{MB}$, and 500 image pairs are taken for every operation. The electronic synchronizer consists of a phase-lock signal coordinator and a laser doublepulse timing controller. The phase-lock signal coordinator receives series of photoelectric switch signals from an encoder, which is a reflective sticker on the hub of the fan. The encoder has a resolution of 360 pulses/ revolution. The output signals of the phase-lock coordinator are fed to the laser double-pulse timing controller according to the settings. The laser double-pulse timing controller bears the output signals for controlling timings for lamp flashing and Q-switching of the lasers as well as the shuttering of the camera.

There are two sub-systems used in the PIV post- processing system: the image interrogation and post interrogation system. By using the cross-correlation technique, two consecutive image frames can be analyzed and developed [8]. This technique is imbedded in the software "VidPIV4" developed by Optical Flow Systems. It calculates the average displacement of local particle groups in these consecutive images. The interrogation window is set to $32 \times 32$ pixels. In order to reduce velocity bias in the regions with large velocity gradients, the ratio of the displacement of the doubleexposed images to the length of the interrogation area is held to be lower than $1 / 4$, as suggested by Keane and Adrian [7]. Filtering and interpolation can identify outliers and then regenerate the missed values. The global filter finds all the globally inconsistent values, while the local filter finds those that may be globally consistent but are not smoothly consistent with the local variations in vector magnitude and direction. The method of interpolation is based on a weighted mean technique that can replace the values at filtered sites in an iterative manner by replacing those with the most surrounding valid values first, working toward those that are less favorably positioned, for accurate and reliable interpolation. Adaptive cross-correlation employing smaller interrogation size and smaller grid spacing is finally applied to the vector field generated from the regular procedure of cross correlation, filtering (global and local), and interpolation to provide higher resolution and accuracy than the first pass. A series of instantaneous vector fields are required to calculate the ensemble averages. By combining a sufficient number of vector fields, the mean velocity field is obtained. In this study, the mean velocity field of the centrifugal flow fan is obtained from the ensemble average of 500 instantaneous image pairs.

\section{Fan performance tests}

The configurations and specifications of the centrifugal flow fans are shown in Figure 2, and the geometric parameters are tabulated in Table 1 . The experimental facilities used in this paper are shown schematically in Figure 3, and they are similar to those used by Yen and Lin [18]. The centrifugal flow fan is clamped laterally in the test section of the AMCA 210 flow bench (ANSI/AMCA Standard 210-85, 1985) for measuring the flow rates. The fresh air is inhaled into the test section by a suction fan, which is attached to the AMCA 210 flow bench. Three different sizes of metal mesh are set at the upstream and downstream of the nozzles to regulate the flow. By averaging the static pressures measured from the plenum, the flow rate can be calculated by the pressure difference. The input power can be found by recording the input currents and voltages. 


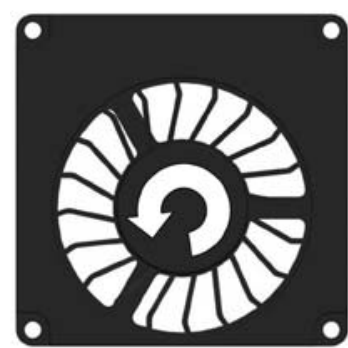

(a) Top view

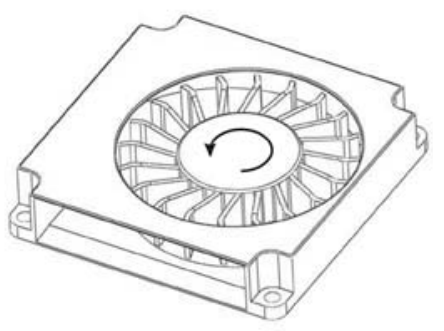

(b) Detailed configuration
Fig. 2. Centrifugal fan (a) top view; (b) detailed configuration.
Table 1. Fan geometry parameters

\begin{tabular}{lcc}
\hline Fan & Fan-A & Fan-B \\
\hline Dimension $L \times H \times W(\mathrm{~mm})$ & $60 \times 60 \times 11$ & $60 \times 60 \times 11$ \\
Hub diameter $(\mathrm{mm})$ & 24 & 24 \\
Number of blades & 17 & 17 \\
Rotor speed (rpm) at 5V & 3100 & 4000 \\
Hub-to-tip at rotor inlet (mm) & 10 & 10 \\
Blade thickness (mm) & 1 & 1 \\
Tip clearance (mm) & 1 & 1 \\
Chord length (mm) & 9 & 9
\end{tabular}

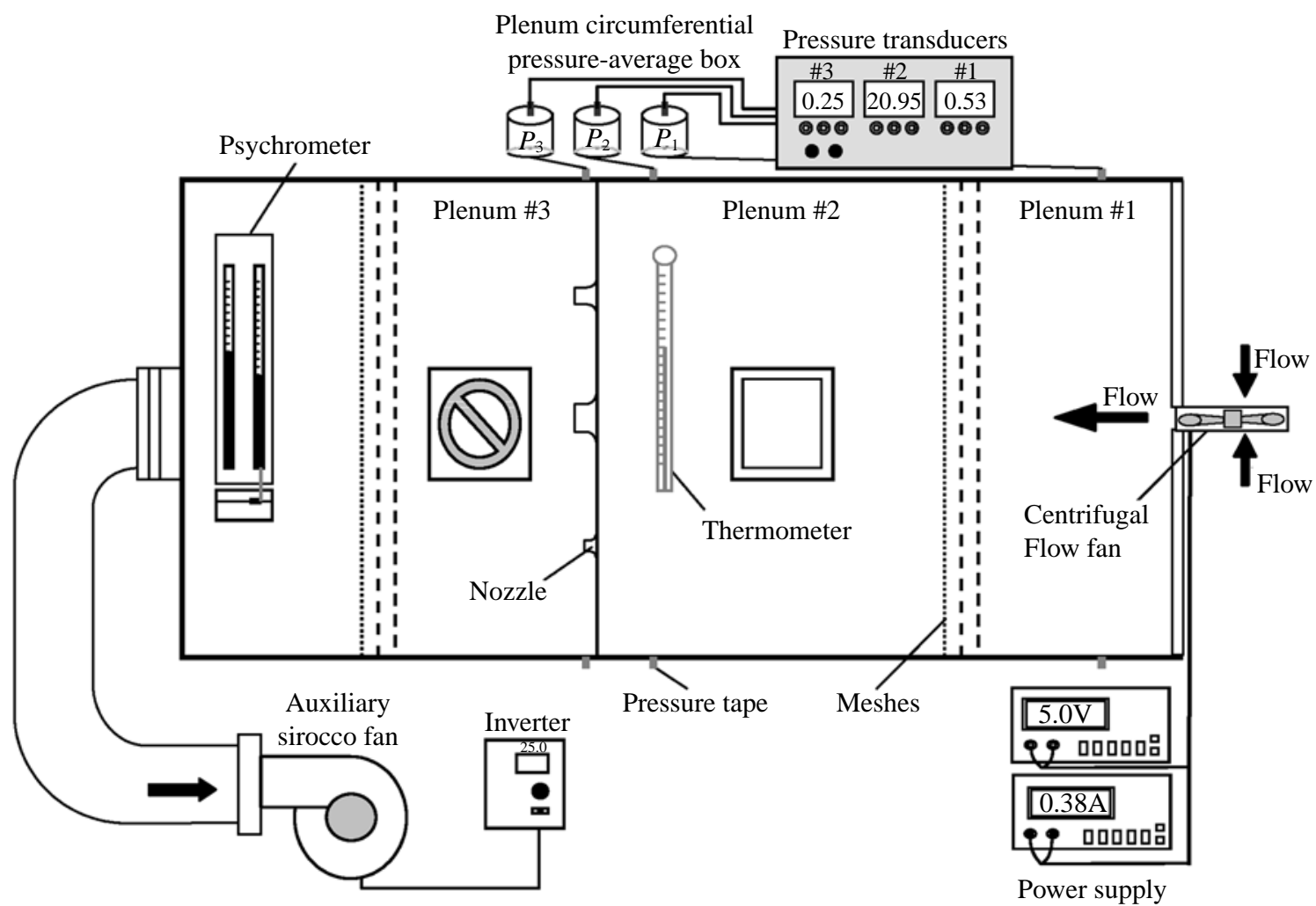

Fig. 3. Schematic of performance experimental facility system setup.

\section{Dimensional analysis in fan}

Dimensional analysis in the centrifugal flow fan has been employed by many researchers $[1,11,17]$. The dimensionless parameters such as head coefficient $\Psi$, flow coefficient $\Phi$, power coefficient $\Lambda$, and the fan efficiency $\eta$ can be derived from the $\Pi$-products theorem and defined as:

$$
\text { Head coefficient } \Psi=\frac{\Delta P_{s}}{\rho N^{2} D^{2}}
$$

$$
\begin{aligned}
& \text { Flow coefficient } \Phi=K \frac{Q}{N D^{3}} \\
& \text { Power coefficient } \Lambda=\frac{\wp}{\rho N^{3} D^{5}}
\end{aligned}
$$

Fan efficiency $\eta=\Phi \Psi / \Lambda$

where $\Delta P_{s}$ is the static pressure rise, $\rho$ is the density of the air, $N$ is the rotor speed, $D$ is the fan diameter, $Q$ is the volume flow rate, $K$ depends on the hub-to-tip ratio [1], and $\wp$ is the fan power. 


\section{RESULTS AND DISCUSSION}

To understand the complex flow phenomena in the centrifugal flow fans, the following procedures are implemented:

(1) Using a PIV measurements system to measure the instantaneous and ensemble average flow field in the downstream.

(2) Discussing the flow field patterns and mean velocity distributions on diametral and lateral planes.

(3) Evaluating the performance coefficients with different flow parameters regulated in the AMCA 210 wind tunnel.

\section{Velocity flow field}

\section{(1)Flows in diametral planes}

The PIV-measured velocity vector field and vorticity contour fields are shown in Figure 4. The streamline patterns are obtained by the shooting method. The vorticity $\Omega_{z}$ is calculated from the mean velocity data by using the central difference scheme, defined as

$$
\Omega_{z}=\frac{\partial V_{y}}{\partial x}-\frac{\partial V_{x}}{\partial y}
$$

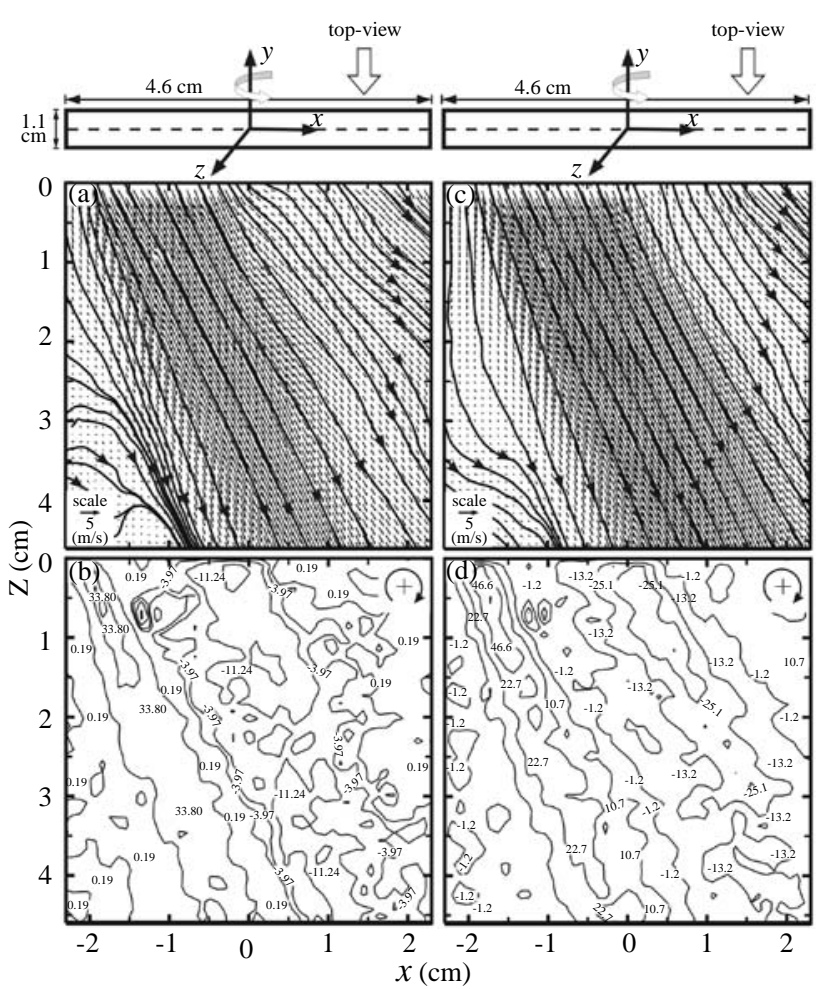

Fig. 4. Flow field pattern (top) and vorticity contour field (bottom) of Fan-A (a, b) and Fan-B (c, d) on the diametral plane. where $V_{x}$ and $V_{y}$ are velocity components in the $x$ and $y$ directions, respectively. The clockwise-rotating vortex is defined as positive. By averaging the 100 pairs of measured instantaneous velocities, Figure 4 shows the velocity vectors and vorticity contours on the diametral plane $(y=0)$ at the near-exit position of Fan-A and FanB, respectively.. As shown in Figure 4(a) and 4(b), the velocity vectors and the vorticity contours move from the left-upper to the right-bottom part of this plot. This phenomenon satisfies the physical model that the impellers rotate counterclockwise. In the left-bottom region of Figure 4(a), the flow velocity is lower due to the lesser influence of the impellers. The maximum vorticity of $33.8 \mathrm{~s}^{-1}$ is observed in Figure 4(b). Figure 4(c) demonstrates the same flow structure as Figure 4(a) except for the higher rotation speed in Fan-B. As the result, the maximum vorticity of $46.6 \mathrm{~s}^{-1}$ is observed in Figure 4(d). Also, the flow direction can be demonstrated in these two panels.

Figure 5 shows the velocity distributions of $V_{x}, V_{z}$, and $|V|$ along $\mathrm{z}$-axis $(x=0 ; y=0)$. In Figure 5(c), the lowest absolute velocity of $1.8 \mathrm{~m} / \mathrm{sec}$ appears at the near-exit position (i.e. $z \sim 0$ ). The highest absolute velocity of $5.6 \mathrm{~m} / \mathrm{s}$ is observed at $z=3 \mathrm{~cm}$. After this point, the velocity decreases as the flow travels farther away. Figure 5(f) shows that the absolute velocity of $7.5 \mathrm{~m} / \mathrm{s}$ of Fan B is larger than the $5.6 \mathrm{~m} / \mathrm{s}$ in Fan-A. The reason is that the induced flow velocity in Fan-B is

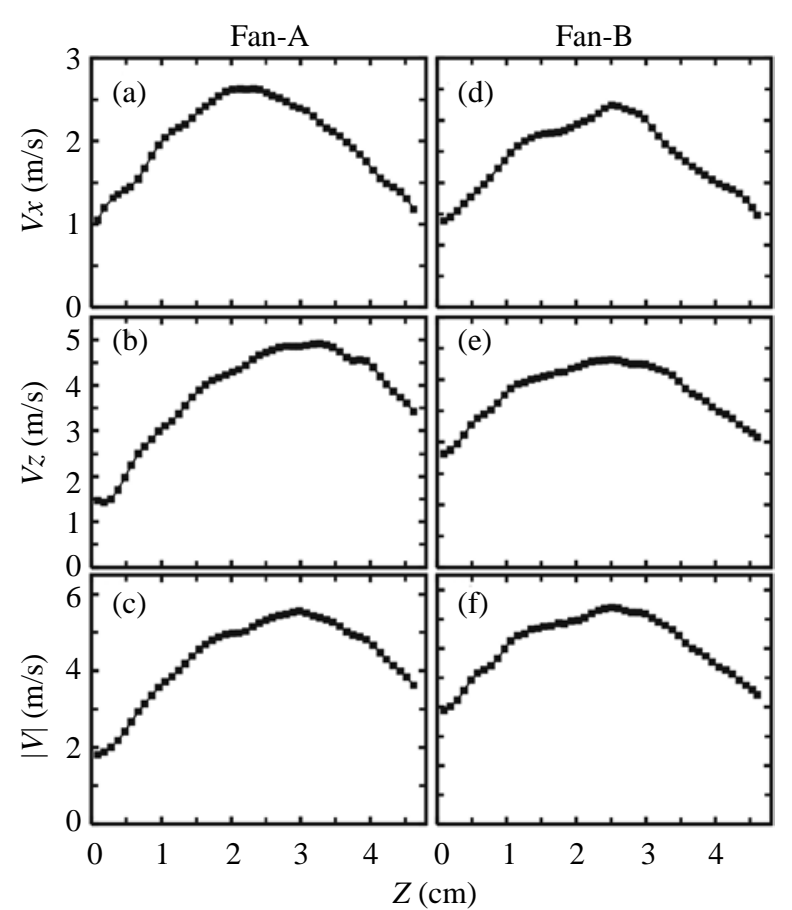

Fig. 5. Distributions of velocity components $(a, b, d, e)$ and absolute velocity (c, f) along z-axis as $x=0, y=0$. 
larger than that in Fan-A. Figure 6 shows the lateral velocity distributions (along the $x$-axis) of $V_{x}, V_{z}$ and $|V|$ on the lateral plane ( $y-z$ plane $)$ at different $\mathrm{z}$ positions, respectively. As shown in Figure 6(a), (b) and (c), the maximum exit velocities of Fan-A decrease as $z$ increases for all curves. In Figure 6(d), (e) and (f), the maximum exit velocities of Fan-B are almost fixed as $0<z<4.6 \mathrm{~cm}$. These plots also show that all the velocity components in Fan-B are larger than in Fan-A due to the higher rotation speed in Fan-B. Experimentally, it is difficult to capture the whole view of the external flow field due to the flow expansion. In this paper, we defined a new modified flow rate $q$ as

$$
q=\frac{Q}{b}=\int v_{z}(x) \cdot d x
$$

where $Q$ is the volume flow rate, $b$ is the width of the capture window, and $v_{z}(x)$ is the velocity profile along the $x$-axis. Using Eq. 8, Figure 7 shows the distribution of the modified flow rate integrated from the data in Figure 6(c) and (f), respectively. It reveals that the maximum modified flow rate $q_{\max }$ appears at the nearexit position. It also shows that as the $z$ position increases, the modified flow rate decreases. This evidences that the flow rate of Fan-B is larger than that of Fan-A.

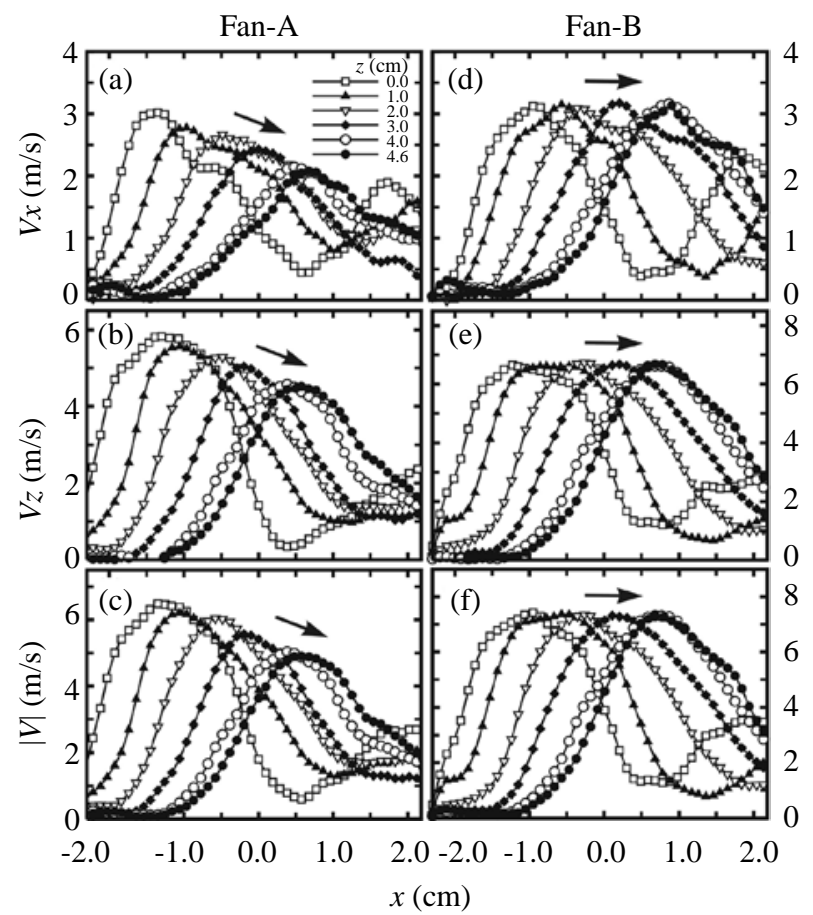

Fig. 6. Distributions of velocity components $(a, b, d, e)$ and absolute velocity $(c, f)$ along the $x$-direction as $y=0$, and $z=0.0,1.0,2.0$, $3.0,4.0,4.6 \mathrm{~cm}$, respectively.

\section{(2) Flows in lateral planes}

Figure 8 shows the distributions of velocity and vorticity on different lateral planes ( $y-z$ plane) of Fan-A (left), and Fan-B (right). Figure 8(a) shows the velocity distribution at $x=0$ on the lateral plane. Resulting from the geometric symmetry, the flow moves downstream symmetrically. Figure 8(b) shows the vorticity contour of Figure $8(\mathrm{a})$; a 0.2 constant vorticity distributes along the z-axis. Figure 8 (c) and (d) show a similar profile to Figure 8(a) and (b) except for the 3.5 constant vorticity.

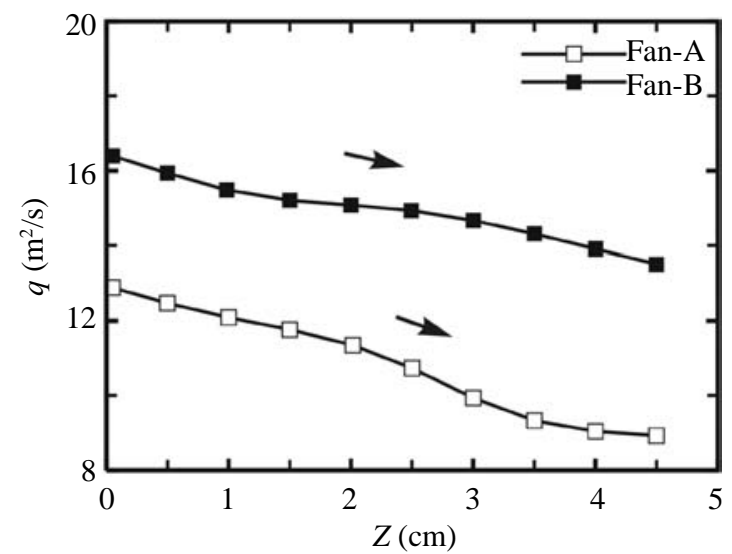

Fig. 7. Modified flow rate as a function of longitudinal position.

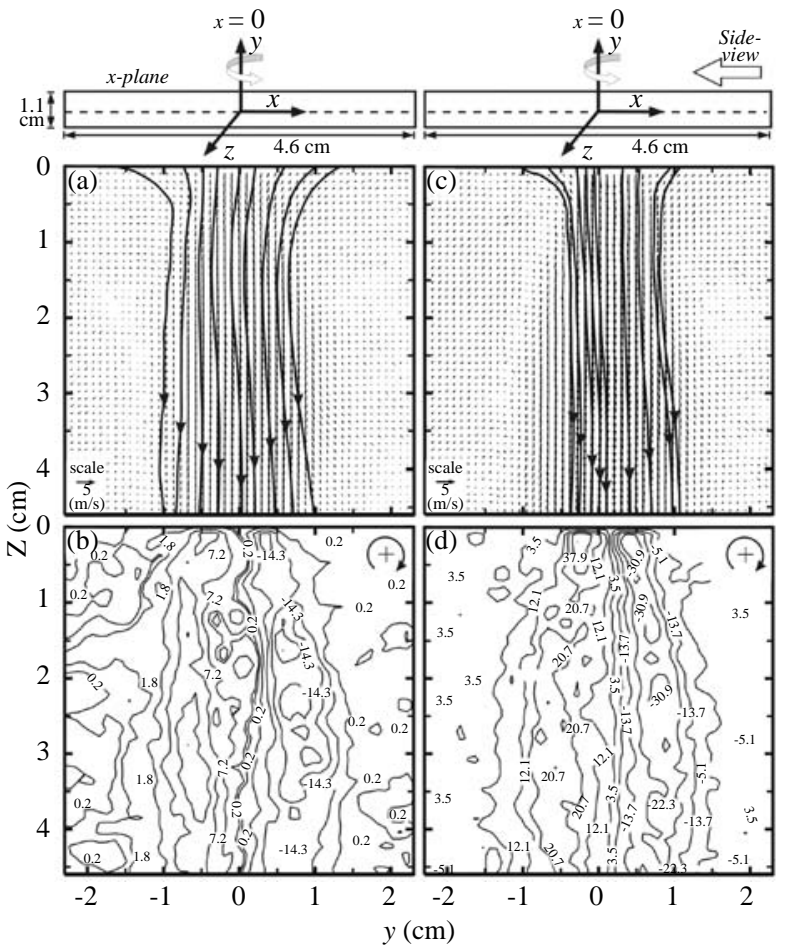

Fig. 8. Flow field pattern (top) and vorticity contour field (bottom) of Fan-A (a, b) and Fan-B (c, d) on the lateral plane. 
Shown in Figure 9 are the downstream velocity distributions of $v_{z}$ along the $z$-axis on different lateral planes. Figure 9(a) shows the velocity distribution of Fan-A. The square notation denotes the velocity distribution at $x=-1.2 \mathrm{~cm}$. It shows the maximum velocity $v_{z}$ of $5.1 \mathrm{~m} / \mathrm{s}$ appearing at $z=1.15 \mathrm{~cm}$ and then decreasing downstream. The solid-circle notation denotes the velocity distribution at $x=0.0 \mathrm{~cm}$. It shows the maximum velocity $v_{z}=4.9 \mathrm{~m} / \mathrm{s}$ appearing at $x=3.0 \mathrm{~cm}$. These results coincide with the data in Figure 5(b). The triangular notation denotes the velocity distribution at $x=+1.2 \mathrm{~cm}$, and the maximum velocity $v_{z}$ is $4.1 \mathrm{~m} / \mathrm{s}$. Figure 9(b) shows the velocity distribution of $v_{z}$ on different lateral planes. It can be observed that all the maximum velocities are larger than those of Fan-A. The solid-circle data can also be checked with Figure 5(e).

\section{Centrifugal fan performance}

The AMCA 210 wind tunnel is used to evaluate the fan performance. To confirm that the flow field is steady, the acquisition system does not capture data until the test system has operated for about 30 seconds. Figure 10(a) shows the performances of the head coefficient with different volume flow rates. The head coefficient of the static pressure is negatively correla-

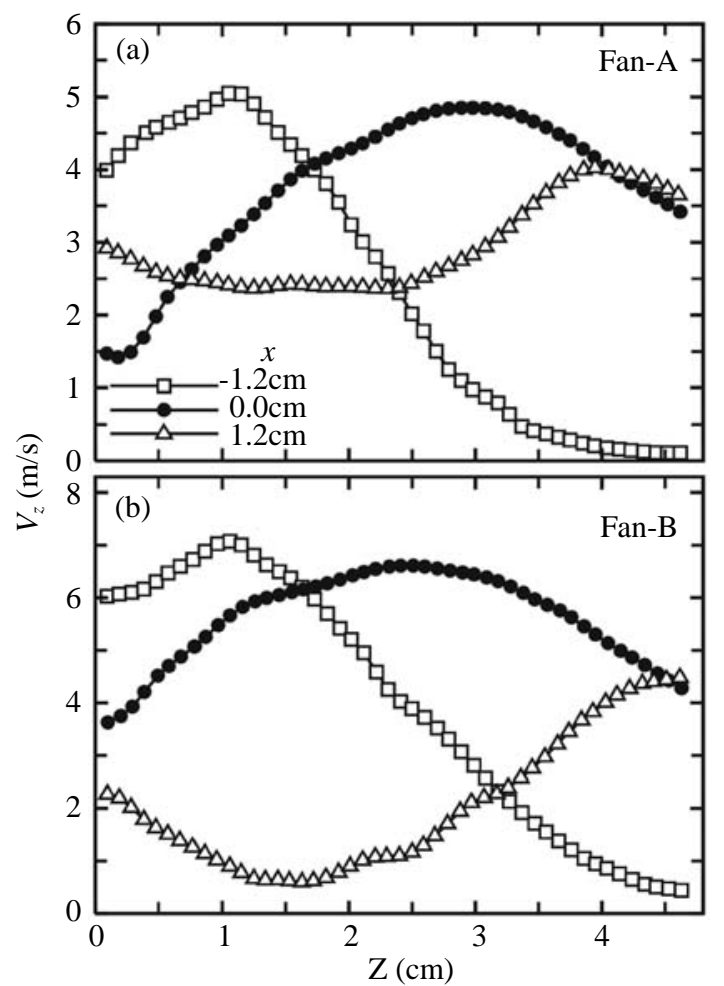

Fig. 9. Velocity component in the $\mathrm{z}$ direction at $y=0$ and $x=-1.2,0.0$, $1.2 \mathrm{~cm}$, respectively. tive to the flow coefficient for these two fans. The head coefficients of Fan-B are larger than those of Fan-A. Figure 10(b) shows the performance curves of the power coefficient with different volume flow rates. These two curves show the positive-linear relationship between the power coefficient and the flow coefficient. This phenomenon presents a serious problem in the centrifugal fan because the motor may overload if the operation point is beyond the designed flow rate. The current investigation provides a basis for that study. Considering the energy consumption, Fan-A consumes more power than Fan-B does. The reason is that Fan-B introduces a higher Reynolds number by rotating at higher speed. This higher Reynolds number conducts a lower drag coefficient, so Fan-B conveys the lower power consumption [2].

In this paper, we present two different rotation speeds of centrifugal fans. The different rotation speeds cause different phenomena in the exit region. These variations certainly influence the efficiency of the fans. Eq. (4) can be use to calculate the fan efficiency, and the results are shown in Figure 11. The efficiency $\eta$ of FanA attains its maximum efficiency of $3.95 \%$ at $\Phi=0.08$. In Fan-B, the maximum efficiency $\eta$ is $13.1 \%$ at $\Phi=$ 0.07 . The extra $9.15 \%$ efficient comes from the rotation speed effect.

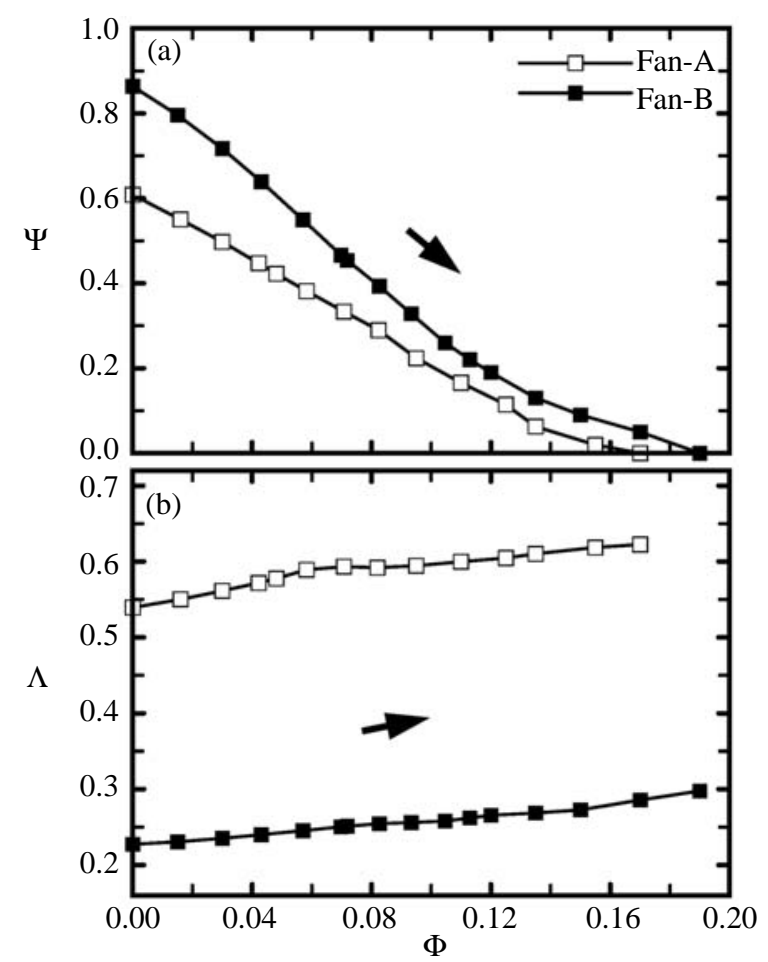

Fig. 10. Plot of head coefficient $\Psi$ (a) and power coefficient $\Lambda$; (b) as a function of the flow coefficient $\Phi$, respectively. 


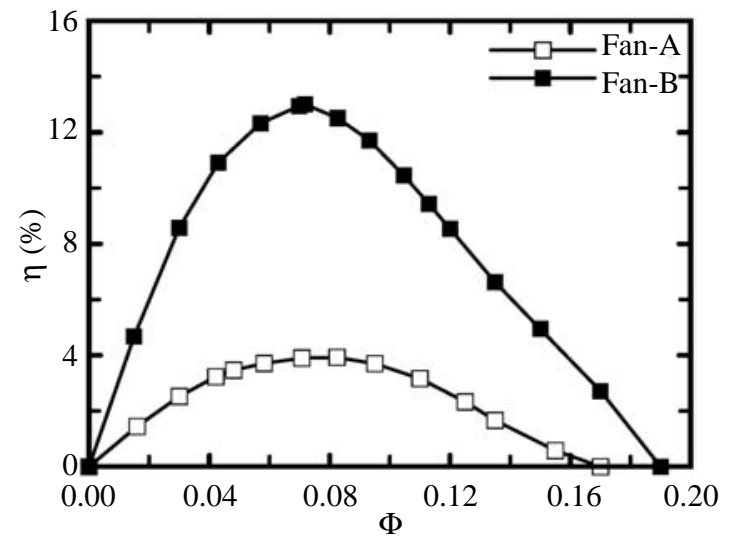

Fig. 11. Plot of fan efficiency $\eta$ as a function of the flow coefficient $\Phi$.

\section{CONCLUSIONS}

In the present work, we experimentally explore two centrifugal fans with identical geometrical structures. These two fans perform at different rotation speeds under the same driving voltage. The PIV method is applied to measure the instantaneous flow fields at the near-exit position. Furthermore, an AMCA 210 flow bench is used to evaluate the performance coefficients. To the best of our knowledge, this is the first paper to discuss the relationship between the near-exit flow patterns and the efficiency parameters. These results can also be applied to the field of optimal design. The following are the characteristics we find:

(1) The lowest absolute flow speed appears at the nearexit position (i.e. $z \approx 0$ ), and the highest absolute speed appears at the point of $z=3 \mathrm{~cm}$. The speed decreases as the longitudinal position moves farther away from the exit.

(2) The maximum modified flow rate appears at the near-exit position. As longitudinal position increases, it decreases.

(3) The efficiency $\eta$ of Fan-A (3100 rpm) attains its maximum of $3.95 \%$ at $\Phi=0.08$. For Fan-B (4000 $\mathrm{rpm})$, the maximum efficiency $\eta$ is $13.1 \%$ at $\Phi=$ 0.07 . The extra $9.15 \%$ efficiency comes from the rotation speed effect.

(4) The higher the rotation speed, the higher the flow rate and the better the efficiency under the conditions of identical geometrical structure and the same driving voltage.

\section{REFERENCES}

1. Bleier, F.P., Fan Handbook, McGraw-Hill Companies Inc., New York, pp. 13-117 (1998).

2. Blevins, R.D., Applied Fluid Dynamics Hankbook, Van Nostrand Reinhold, New York (1984).
3. Chen, P., Nayagen. M.S., Bolton, A.N., and Simpson, H.C., "Unstable Flow in Centrifugal Fans," ASME Journal of Fluids Engineering, Vol. 118, pp. 128-133 (1996).

4. Dilin, P., Sakai, T., Wilson, M., and Whitfield, A., "A Computational and Experimental Evaluation of the Performance of a Centrifugal Fan Volute," Proceedings Institution of Mechanical Engineers, Part A, Journal of Power and Energy, Vol. 212, pp. 235-246 (1998).

5. Eck, B., Fans: Design and Operation of Centrifugal, Axial-Flow and Cross-Flow Fans, Pergamon Press, Oxford, New York (1973).

6. Jeon, W.H., "A Numerical Study on the Effects of Design Parameters on the Performance and Noise of a Centrifugal Fan," Journal of Sound and Vibration, Vol. 265, pp. 221-230 (2003).

7. Keane, R.D. and Adrian, R.J., "Optimization of Particle Image Velocimeters Part I: Double Pulsed Systems," Measurement Science and Technology, Vol. 1, No. 6, pp. 1202-1215 (1990).

8. Keane, R.D. and Adrian, R.J., "Theory of Cross-Correlation Analysis of PIV Images," Applied Scientific Research, Vol. 49, No. 2, pp. 191-215 (1992).

9. Lin, S.C. and Huang, C.L., "An Integrated Experimental and Numerical Study of Forward-Curved Centrifugal Fan," Experimental Thermal and Fluid Science, Vol. 26, pp. 421-434 (2002).

10. Mei, R., "Velocity Fidelity of Flow Tracer Particles," Experiments in Fluid, Vol. 22, No. 1, pp. 1-13 (1996).

11. Oh, K.J. and Kang, S.H., "A Numerical Investigation of the Dual Performance Characteristics of a Small Propeller Fan Using Viscous Flow Calculations," Computers and Fluids, Vol. 28, pp. 815-823 (1999).

12. Raj, D. and Swim, W.B., "Measurements of the Mean Flow Velocity and Velocity Fluctuation at the Exit of a F-C Centrifugal Fan Rotor," Journal of Engineering for Power, Vol. 103, No. 2, pp. 393-399 (1981).

13. Sandra, V.S., Rafael, B.T., Carlos, S.M., and Jose, G.P., "Unsteady Flow Pattern Characteristics Downstream of a Forward-Curved Blades Centrifugal Fan," ASME Journal of Fluids Engineering, Vol. 123, pp. 265-270 (2001).

14. Tusrusaki, H., Tsujimoto, Y., Yoshida, Y., and Kitagawa, K., "Visualizatioin Measurement and Numerical Analysis of Internal Flow in Cross-Flow Fan," ASME Journal of Fluids Engineering, Vol. 119, pp. 633-638 (1997).

15. Tusrusaki, H., Shimizu, H., Tsujimoto, Y., Yoshida, Y., and Kitagawa, K., "Study of Cross-Flow-Fan Internal Flow by Flow Visualization," JSME International Journal, Series B, Vol. 39, No. 3, pp. 540-545 (1996).

16. Wallis, R.A., Axial Flow Fans and Ducts, John Wiley \& Sons Inc., New York (1983).

17. Wright, T., Fluid Machinery: Performance, Analysis, and Design, CRC Press, Boca Raton, FL (1999). 
18. Yen, S.C. and Lin, K.T., "Exit Flow Field and Performance of Axial Flow Fans," ASME Journal of Fluids Engineering, Vol. 128, March, pp.332-340 (2006).

\section{NOMENCLATURE}

$D \quad$ fan diameter

$D_{H u b}$ hub diameter of fans

$N$ rotor speed

$P_{s} \quad$ static pressure

$Q \quad$ volume flow rate

$q$ modified flow rate

$t \quad$ maximum thickness of the blade

$x, y, z$ Cartesian coordinates with origin at the center of fans
$V_{x} \quad$ horizontal velocity component in $x$ directions, $\mathrm{cm} / \mathrm{s}$

$V_{y} \quad$ vertical velocity component in $y$ directions, $\mathrm{cm} / \mathrm{s}$

$V_{z} \quad$ longitudinal velocity component in $z$ directions, $\mathrm{cm} / \mathrm{s}$

$|V| \quad$ absolute velocity

$\rho$ density of the air

$\Omega \quad$ vorticity, $1 / \mathrm{s}$

$\Psi \quad$ head coefficient, $\left(=\Delta P_{s} /\left(\rho N^{2} D^{2}\right)\right)$

$\Lambda \quad$ power coefficient $\left(=\wp /\left(\rho N^{3} D^{5}\right)\right)$

$\Phi$ flow coefficient $\left(=K Q / N D^{3}\right)$

$\eta \quad$ fan efficiency $(=\Psi \Phi / \Lambda)$

$K \quad$ constant depending on the hub-to-tip

$\wp \quad$ fan power 